\title{
Solutions to Riemann-Liouville fractional integrodifferential equations via fractional resolvents
}

\author{
Shaochun $\mathrm{Ji}^{1^{*}}$ (D) and Dandan Yang ${ }^{2}$
}

\section{"Correspondence:}

jiscmath@163.com

${ }^{1}$ Faculty of Mathematics and

Physics, Huaiyin Institute of

Technology, Huaian, P.R. China

Full list of author information is

available at the end of the article

\section{Springer}

\begin{abstract}
This paper is concerned with the semilinear fractional integrodifferential system with Riemann-Liouville fractional derivative. Firstly, we introduce the suitable $C_{1-\alpha}$-solution to Riemann-Liouville fractional integrodifferential equations in the new frame of fractional resolvents. Some properties of fractional resolvents are given. Then we discuss the sufficient conditions for the existence of solutions without the Lipschitz assumptions to nonlinear item. Finally, an example on fractional partial differential equations is presented to illustrate our abstract results.
\end{abstract}

MSC: $34 \mathrm{~A} 08 ; 47 \mathrm{~A} 10$

Keywords: Fractional resolvents; Riemann-Liouville fractional derivative; Integrodifferential equations; Fixed point theorems

\section{Introduction}

Fractional differential equations have received much attention over the past two decades, as they are found to be important models in many physical, biological, and engineering problems. In fact, they can be regarded as alternative models to nonlinear differential equations and many physical phenomena with memory characteristics can be described by fractional differential equations; see, for instance, [1-7]. Recently, the theories of fractional differential equations with classical Caputo and Riemann-Liouville derivative have been developed and some basic properties are obtained including existence and controllability, see [8-27]. Among them, the differential equations with Caputo fractional derivative are studied extensively. By probability density functions, Wang and Zhou [13] gave a suitable concept of mild solutions to Caputo fractional evolution equations. Balachandran and Kiruthika [11], Balasubramaniam and Tamilalagan [23] proved the existence of solutions to Caputo fractional integrodifferential equations by using resolvent operators. Mallika and Baleanu et al. [26] studied the fractional neutral integrodifferential equation with nonlocal conditions by fixed point theorems and resolvent operators.

On the other hand, in the papers of Heymans and Podlubny [28], Agarwal et al. [29], Baleanu et al. [30], it was shown that Riemann-Liouville fractional differential equations are useful in physics to model viscoelasticity and have different properties from the Ca-

(c) The Author(s) 2019. This article is licensed under a Creative Commons Attribution 4.0 International License, which permits use, sharing, adaptation, distribution and reproduction in any medium or format, as long as you give appropriate credit to the original author(s) and the source, provide a link to the Creative Commons licence, and indicate if changes were made. The images or other third party material in this article are included in the article's Creative Commons licence, unless indicated otherwise in a credit line to the material. If material is not included in the article's Creative Commons licence and your intended use is not permitted by statutory regulation or exceeds the permitted use, you will need to obtain permission directly from the copyright holder. To view a copy of this licence, visit http://creativecommons.org/licenses/by/4.0/. 
puto derivative. As the Riemann-Liouville fractional derivative has a singularity at zero, the mathematical analysis to Riemann-Liouville fractional differential equations is more complicated. In this paper we will consider the following semilinear integrodifferential system with a Riemann-Liouville fractional derivative:

$$
\left\{\begin{array}{l}
D^{\alpha} x(t)=A x(t)+f\left(t, x(t), \int_{0}^{t} h(t, s, x(s)) \mathrm{d} s\right), \quad t \in J^{\prime}:=(0, b] \\
\lim _{t \rightarrow 0^{+}} \Gamma(\alpha) t^{1-\alpha} x(t)=x_{0},
\end{array}\right.
$$

where $0<\alpha<1, D^{\alpha}$ is the Riemann-Liouville fractional derivative of order $\alpha, A: D(A) \subseteq$ $X \rightarrow X$ is the infinitesimal generator of an order- $\alpha$ fractional resolvent $\left\{S_{\alpha}(t), t>0\right\}$ on a Banach space $X$, the operators $h: \Delta \times X \rightarrow X, f: J \times X \times X \rightarrow X$ are nonlinear functions, where $\Delta=\{(t, s), 0 \leq s \leq t \leq b\}, J:=[0, b]$.

Some authors have discussed the solutions to fractional differential equations with Riemann-Liouville fractional derivative [18, 31-33]. For the mild solution, there are two different types of representation that have been given. The first one was constructed in terms of a probability density function. By Laplace transformation and probability density function, Liu and $\mathrm{Li}$ [31] gave an appropriate concept of solutions to a semilinear differential system when $A$ generates a $C_{0}$-semigroup. The second one was presented in terms of fractional resolvents. In [32], based on $(\alpha, k)$-regularized operators, Lizama got the representation of solutions for linear fractional order differential equations. Using order- $\alpha$ resolvents, Li and Peng [21], Fan [33] discussed the solutions to fractional homogeneous and inhomogeneous linear differential system, respectively. As is well known, $C_{0}$-semigroup is a useful tool in the study of first order differential equations in Banach spaces. In a similar way, fractional resolvents play an important role in the theory of fractional integrodifferential equations. For a compact $C_{0}$-semigroup $T(t)$, it is continuous in the sense of operator norm for $t>0$. Then it is a natural question to ask whether the result is valid in the case of Riemann-Liouville fractional resolvents; see Lemma 2.5. This is one motivation of this paper.

Recently some interesting results on Caputo fractional resolvents have been given in $[11,12,26]$. We note that the properties of resolvent operators for Caputo derivative and Riemann-Liouville derivative are different in essence, though neither of them has the semigroup property. For Caputo fractional resolvents $T_{\alpha}(t), T_{\alpha}(0) x=x$ for every $x \in X$, but it is not valid in the case of Riemann-Liouville fractional resolvents. So another motivation of this paper is to formulate the suitable solution to problem (1.1) by RiemannLiouville fractional resolvents in a Banach space $C_{1-\alpha}(J, X)$, which is constructed to solve the difficulty of fractional resolvents' unboundedness at $t=0$. Then without the Lipschitz conditions, the existence of solutions to problem (1.1) is discussed.

The paper is organized as follows. In Sect. 2, we recall some concepts and facts about the fractional resolvents. Section 3 is devoted to the sufficient conditions for solutions to problem (1.1). Finally, an example is presented to illustrate the application of our results.

\section{Preliminaries}

We denote by $C(J, X)$ the space of $X$-valued continuous functions on $J$ with the norm $\|x\|=\sup \{\|x(t)\|, t \in J\}, B(X)$ the space of all bounded linear operators from $X$ to itself, $L^{p}(J, X)$ the space of $X$-valued Bochner integrable functions with the norm $\|f\|_{L^{p}}=$ 
$\left(\int_{0}^{b}\|f(t)\|^{p} \mathrm{~d} t\right)^{\frac{1}{p}}$. In order to define the solution to problem (1.1), we consider the space

$$
C_{1-\alpha}(J, X):=\left\{x(\cdot): \cdot^{1-\alpha} x(\cdot) \in C(J, X), 0<\alpha<1\right\}
$$

with the norm $\|x\|_{C_{1-\alpha}}=\sup \left\{\left\|t^{1-\alpha} x(t)\right\|_{X}: t \in J\right\}$, where $\left.t^{1-\alpha} x(t)\right|_{t=0}=\lim _{t \rightarrow 0^{+}} t^{1-\alpha} x(t)$. Obviously $C_{1-\alpha}(J, X)$ is a Banach space.

Now we recall some definitions and results on fractional derivative and fractional differential equations.

Definition 2.1 ([3]) The Riemann-Liouville fractional integral of a function $f \in L^{1}(J, X)$ of order $\alpha \in \mathbb{R}^{+}$is defined by

$$
I_{t}^{\alpha} f(t)=\frac{1}{\Gamma(\alpha)} \int_{0}^{t}(t-s)^{\alpha-1} f(s) \mathrm{d} s, \quad t>0
$$

where $\Gamma(\cdot)$ is the gamma function.

Definition 2.2 ([3]) The Riemann-Liouville fractional order derivative of order $\alpha \in \mathbb{R}^{+}$ of a function $f \in L^{1}(J, X)$ is defined by

$$
D^{\alpha} f(t)=\frac{1}{\Gamma(n-\alpha)} \frac{\mathrm{d}^{n}}{\mathrm{~d} t^{n}} \int_{0}^{t}(t-s)^{n-\alpha-1} f(s) \mathrm{d} s, \quad t>0,
$$

where $\alpha \in(n-1, n], n \in \mathbb{N}$.

Especially for $0<\alpha<1, D^{\alpha} f(t)=\frac{1}{\Gamma(1-\alpha)} \frac{\mathrm{d}}{\mathrm{d} t} \int_{0}^{t}(t-s)^{-\alpha} f(s) \mathrm{d} s, t>0$.

Let the symbol $*$ be the convolution $(f * g)(t)=\int_{0}^{t} f(t-s) g(s) \mathrm{d} s$. For the sake of convenience, we take $g_{\alpha}(t):=\frac{t^{\alpha-1}}{\Gamma(\alpha)}$ for $t>0$ and $g_{\alpha}(t)=0$ for $t \leq 0$. Then, for $0<\alpha<1$,

$$
I_{t}^{\alpha} f(t)=\left(g_{\alpha} * f\right)(t), \quad D^{\alpha} f(t)=\frac{\mathrm{d}}{\mathrm{d} t}\left(g_{1-\alpha} * f\right)(t) .
$$

Definition 2.3 ([21]) Let $0<\alpha<1$. A family $\left\{S_{\alpha}(t), t>0\right\} \subseteq B(X)$ is called an order- $\alpha$ fractional resolvent if it satisfies the following assumptions:

(a) $S_{\alpha}(\cdot) x \in C\left(\mathbb{R}^{+}, X\right)$ and $\lim _{t \rightarrow 0^{+}} \Gamma(\alpha) t^{1-\alpha} S_{\alpha}(t) x=x, x \in X$;

(b) $S_{\alpha}(t) S_{\alpha}(s)=S_{\alpha}(s) S_{\alpha}(t), s, t>0$;

(c) $S_{\alpha}(t) I_{s}^{\alpha} S_{\alpha}(s)-I_{t}^{\alpha} S_{\alpha}(t) S_{\alpha}(s)=g_{\alpha}(t) I_{s}^{\alpha} S_{\alpha}(s)-g_{\alpha}(s) I_{t}^{\alpha} S_{\alpha}(t), s, t>0$.

The linear operator $A$ defined by

$$
A x=\Gamma(2 \alpha) \lim _{t \rightarrow 0^{+}} \frac{t^{1-\alpha} S_{\alpha}(t) x-\frac{1}{\Gamma(\alpha)} x}{t^{\alpha}}, \quad x \in D(A),
$$

is the infinitesimal generator of the fractional resolvent $S_{\alpha}(t)$, where

$$
D(A)=\left\{x \in X: \lim _{t \rightarrow 0+} \frac{t^{1-\alpha} S_{\alpha}(t) x-\frac{1}{\Gamma(\alpha)} x}{t^{\alpha}} \text { exists }\right\} .
$$

Note that the fractional resolvent $S_{\alpha}(t)$ is unbounded when $t$ is sufficiently small, but $t^{1-\alpha} S_{\alpha}(t)$ is bounded on $J=[0, b]$. We denote $M=\sup _{t \in J}\left\|t^{1-\alpha} S_{\alpha}(t)\right\|$. 
Lemma 2.4 ([21]) Let $\left\{S_{\alpha}(t), t>0\right\}$ be an order- $\alpha$ fractional resolvent and $A$ be its infinitesimal generator. Then:

(a) $S_{\alpha}(t) x \in D(A)$ and $A S_{\alpha}(t) x=S_{\alpha}(t) A x$ for all $x \in D(A), t>0$.

(b) For all $x \in X, t>0, S_{\alpha}(t) x=\frac{t^{\alpha-1}}{\Gamma(\alpha)} x+A I_{t}^{\alpha} S_{\alpha}(t) x$.

(c) For all $x \in D(A), t>0, S_{\alpha}(t) x=\frac{t^{\alpha-1}}{\Gamma(\alpha)} x+I_{t}^{\alpha} S_{\alpha}(t) A x$.

(d) $A$ is closed and densely defined.

As fractional resolvents do not satisfy the property of semigroups, we need the following convergence results for resolvents in the uniform operator topology.

Lemma 2.5 Let $\left\{t^{1-\alpha} S_{\alpha}(t), t>0\right\}$ be equicontinuous and compact. Then, for every $t>0$,

(a) $\lim _{h \rightarrow 0^{+}}\left\|(t+h)^{1-\alpha} S_{\alpha}(t+h)-\Gamma(\alpha) h^{1-\alpha} S_{\alpha}(h) \cdot t^{1-\alpha} S_{\alpha}(t)\right\|=0$;

(b) $\lim _{h \rightarrow 0^{+}}\left\|t^{1-\alpha} S_{\alpha}(t)-\Gamma(\alpha) h^{1-\alpha} S_{\alpha}(h) \cdot(t-h)^{1-\alpha} S_{\alpha}(t-h)\right\|=0$.

Proof As $t^{1-\alpha} S_{\alpha}(t)$ is compact for $t>0$, we have the set

$$
P_{t}=\left\{t^{1-\alpha} S_{\alpha}(t) x:\|x\| \leq 1\right\},
$$

is precompact in $X$ for every $t>0$. Then we can find a finite family $\left\{t^{1-\alpha} S_{\alpha}(t) x_{i}:\left\|x_{i}\right\| \leq\right.$ $1\}_{i=1}^{m} \subset P_{t}$ satisfying for every $x,\|x\| \leq 1$, there exists $x_{i}, i=1, \ldots, m$, such that

$$
\left\|t^{1-\alpha} S_{\alpha}(t) x-t^{1-\alpha} S_{\alpha}(t) x_{i}\right\|<\frac{\varepsilon}{3(1+\Gamma(\alpha) M)} .
$$

From Definition 2.3(a), there exists $h_{1}>0$ such that

$$
\left\|t^{1-\alpha} S_{\alpha}(t) x_{i}-\Gamma(\alpha) h^{1-\alpha} S_{\alpha}(h) \cdot t^{1-\alpha} S_{\alpha}(t) x_{i}\right\|<\frac{\varepsilon}{3},
$$

for every $0<h \leq h_{1}$ and $1 \leq i \leq m$.

Moreover, as $t^{1-\alpha} S_{\alpha}(t)$ is equicontinuous for $t>0$, we can find $h_{2}>0$ such that

$$
\left\|(t+h)^{1-\alpha} S_{\alpha}(t+h) x-t^{1-\alpha} S_{\alpha}(t) x\right\|<\frac{\varepsilon}{3},
$$

for every $0<h \leq h_{2}$ and $\|x\| \leq 1$.

Now for $0<h \leq \min \left\{h_{1}, h_{2}\right\}$ and $\|x\| \leq 1$, it follows from (2.1)-(2.3) that

$$
\begin{aligned}
\|( & +h)^{1-\alpha} S_{\alpha}(t+h) x-\Gamma(\alpha) h^{1-\alpha} S_{\alpha}(h) \cdot t^{1-\alpha} S_{\alpha}(t) x \| \\
\leq & \left\|(t+h)^{1-\alpha} S_{\alpha}(t+h) x-t^{1-\alpha} S_{\alpha}(t) x\right\| \\
& +\left\|t^{1-\alpha} S_{\alpha}(t) x-t^{1-\alpha} S_{\alpha}(t) x_{i}\right\| \\
& +\left\|t^{1-\alpha} S_{\alpha}(t) x_{i}-\Gamma(\alpha) h^{1-\alpha} S_{\alpha}(h) \cdot t^{1-\alpha} S_{\alpha}(t) x_{i}\right\| \\
& +\left\|\Gamma(\alpha) h^{1-\alpha} S_{\alpha}(h) \cdot t^{1-\alpha} S_{\alpha}(t) x_{i}-\Gamma(\alpha) h^{1-\alpha} S_{\alpha}(h) \cdot t^{1-\alpha} S_{\alpha}(t) x\right\| \\
< & \frac{\varepsilon}{3}+\frac{\varepsilon}{3(1+\Gamma(\alpha) M)}+\frac{\varepsilon}{3} \\
& +\Gamma(\alpha))\left\|h^{1-\alpha} S_{\alpha}(h)\left[t^{1-\alpha} S_{\alpha}(t) x_{i}-t^{1-\alpha} S_{\alpha}(t) x\right]\right\| \\
\leq & \frac{\varepsilon}{3}+\frac{\varepsilon}{3(1+\Gamma(\alpha) M)}+\frac{\varepsilon}{3}+\Gamma(\alpha) M \frac{\varepsilon}{3(1+\Gamma(\alpha) M)} \\
\leq & \varepsilon
\end{aligned}
$$


which implies that, for every $t>0$,

$$
\lim _{h \rightarrow 0}\left\|(t+h)^{1-\alpha} S_{\alpha}(t+h)-\Gamma(\alpha) h^{1-\alpha} S_{\alpha}(h) \cdot t^{1-\alpha} S_{\alpha}(t)\right\|=0 .
$$

(b) Let $t>0$ and $0<h<\min \{t, b\}$. Then, for $\|x\| \leq 1$, we have

$$
\begin{aligned}
& \left\|t^{1-\alpha} S_{\alpha}(t) x-\Gamma(\alpha) h^{1-\alpha} S_{\alpha}(h) \cdot(t-h)^{1-\alpha} S_{\alpha}(t-h) x\right\| \\
& =\left\|t^{1-\alpha} S_{\alpha}(t) x-(t+h)^{1-\alpha} S_{\alpha}(t+h) x\right\| \\
& \quad+\left\|(t+h)^{1-\alpha} S_{\alpha}(t+h) x-\Gamma(\alpha) h^{1-\alpha} S_{\alpha}(h) \cdot t^{1-\alpha} S_{\alpha}(t) x\right\| \\
& \quad+\left\|\Gamma(\alpha) h^{1-\alpha} S_{\alpha}(h) \cdot\left[t^{1-\alpha} S_{\alpha}(t) x-(t-h)^{1-\alpha} S_{\alpha}(t-h) x\right]\right\| \\
& \leq \| \\
& \quad \\
& \quad+\left\|(t+h)^{1-\alpha} S_{\alpha}(t) x-(t+h)^{1-\alpha} S_{\alpha}(t+h) x\right\| \\
& \quad+\Gamma(\alpha) M\left\|t^{1-\alpha} S_{\alpha}(t) x-(t-h)^{1-\alpha} S_{\alpha}(t-h) x\right\|,
\end{aligned}
$$

which implies the corresponding result by the conclusion of Lemma 2.5(a) and the equicontinuity of $\left\{t^{1-\alpha} S_{\alpha}(t), t>0\right\}$.

Definition 2.6 A function $x \in C_{1-\alpha}(J, X)$ is called a solution to problem (1.1) if it satisfies

$$
x(t)=\frac{t^{\alpha-1}}{\Gamma(\alpha)} x_{0}+A I_{t}^{\alpha} x(t)+I_{t}^{\alpha} f\left(t, x(t), \int_{0}^{t} h(t, s, x(s)) \mathrm{d} s\right), \quad t \in J^{\prime} .
$$

Lemma 2.7 ([34]) Let $f \in L^{p}(J, X)$ with $1 \leq p<\infty$. Then

$$
\lim _{h \rightarrow 0} \int_{0}^{b}\|f(t+h)-f(t)\|^{p} \mathrm{~d} t=0
$$

where $f(t)=0$ for $t \neq J$.

\section{Main results}

In this section we shall discuss the concept of solution to problem (1.1) by fractional resolvent method and give its existence theorem without Lipschitz assumptions to nonlinear item $f$. Let $r$ be a finite positive constant and set $W_{r}=\left\{x \in C_{1-\alpha}(J, X):\|x\|_{C_{1-\alpha}} \leq r\right\}$. For brevity, we define the integral operator $H$ by $(H x)(t)=\int_{0}^{t} h(t, s, x(s)) \mathrm{d} s, x \in C_{1-\alpha}(J, X)$. We give the following hypotheses on fractional integrodifferential system (1.1).

(H1) $\left\{t^{1-\alpha} S_{\alpha}(t), t>0\right\}$ is equicontinuous and compact.

(H2) The function $h: \Delta \times X \rightarrow X$ satisfies the following:

(1) For a.e. $(t, s) \in \Delta$, the function $h(t, s, \cdot): X \rightarrow X$ is continuous and for all $x \in X$, the function $h(\cdot, \cdot, x): \Delta \rightarrow X$ is strongly measurable;

(2) There exists $m \in \mathbb{R}^{+}$such that $\|h(t, s, x)\| \leq m\|x\|$.

(H3) The function $f: J \times X \times X \rightarrow X$ satisfies the following:

(1) $f(t, \cdot, \cdot)$ is continuous for a.e. $t \in[0, b]$ and $f(\cdot, x, y):[0, b] \rightarrow X$ is measurable for all $x, y \in X$; 
(2) For a.e. $t \in[0, b]$ and $x, y \in X$,

$$
\begin{gathered}
\|f(t, x, y)\| \leq \theta(t)+\rho t^{1-\alpha}(\|x\|+\|y\|) \\
\text { where } \theta(t) \in L^{p}(J, X), p>\frac{1}{\alpha} \text { and } 0<\rho<\frac{\alpha^{2}}{M b \alpha+M b^{2} m}
\end{gathered}
$$

Lemma 3.1 Let $f \in L^{p}(J, X), p>\frac{1}{\alpha}$ and hypothesis (H1) be satisfied. Then the convolution

$$
\left(S_{\alpha} * f\right)(t)=\int_{0}^{t} S_{\alpha}(t-s) f(s) \mathrm{d} s, \quad t \in J^{\prime},
$$

exists and defines a continuous function on $J^{\prime}$.

Proof From Proposition 1.3.4 in [35], we know that $S_{\alpha}(t-\cdot) f(\cdot)$ is measurable on $(0, t)$. Moreover, we have

$$
\begin{aligned}
\left\|\left(S_{\alpha} * f\right)(t)\right\| & =\left\|\int_{0}^{t}\left((t-s)^{1-\alpha} S_{\alpha}(t-s)\right) \cdot(t-s)^{\alpha-1} f(s) \mathrm{d} s\right\| \\
& \leq M \int_{0}^{t}\left\|(t-s)^{\alpha-1} f(s)\right\| \mathrm{d} s \\
& \leq M\|f\|_{L^{p}}\left(\int_{0}^{t}\left((t-s)^{\alpha-1}\right)^{\frac{p}{p-1}} \mathrm{~d} s\right)^{\frac{p-1}{p}} \\
& \leq M\|f\|_{L^{p}}\left(\left(\frac{p-1}{\alpha p-1}\right) t^{\frac{\alpha p-1}{p-1}}\right)^{\frac{p-1}{p}} \\
& \leq M\|f\|_{L^{p}} b^{\alpha-\frac{1}{p}}\left(\frac{p-1}{\alpha p-1}\right)^{1-\frac{1}{p}}
\end{aligned}
$$

which shows that $S_{\alpha} * f$ exists.

Next we show that $S_{\alpha} * f \in C\left(J^{\prime}, X\right)$. Let $0<\varepsilon<t_{1}<t_{2} \leq b$, then we have

$$
\begin{aligned}
& \left\|\left(S_{\alpha} * f\right)\left(t_{2}\right)-\left(S_{\alpha} * f\right)\left(t_{1}\right)\right\| \\
& =\left\|\int_{0}^{t_{2}} S_{\alpha}\left(t_{2}-s\right) f(s) \mathrm{d} s-\int_{0}^{t_{1}} S_{\alpha}\left(t_{1}-s\right) f(s) \mathrm{d} s\right\| \\
& \leq\left\|\int_{0}^{t_{1}-\varepsilon}\left[\left(t_{2}-s\right)^{1-\alpha} S_{\alpha}\left(t_{2}-s\right)-\left(t_{1}-s\right)^{1-\alpha} S_{\alpha}\left(t_{1}-s\right)\right] \cdot\left(t_{2}-s\right)^{\alpha-1} f(s) \mathrm{d} s\right\| \\
& \quad+\left\|\int_{t_{1}-\varepsilon}^{t_{1}}\left[\left(t_{2}-s\right)^{1-\alpha} S_{\alpha}\left(t_{2}-s\right)-\left(t_{1}-s\right)^{1-\alpha} S_{\alpha}\left(t_{1}-s\right)\right] \cdot\left(t_{2}-s\right)^{\alpha-1} f(s) \mathrm{d} s\right\| \\
& \quad+\left\|\int_{0}^{t_{1}}\left(t_{1}-s\right)^{1-\alpha} S_{\alpha}\left(t_{1}-s\right) \cdot\left[\left(t_{2}-s\right)^{\alpha-1}-\left(t_{1}-s\right)^{\alpha-1}\right] f(s) \mathrm{d} s\right\| \\
& \quad+\left\|\int_{t_{1}}^{t_{2}}\left(t_{2}-s\right)^{1-\alpha} S_{\alpha}\left(t_{2}-s\right) \cdot\left(t_{2}-s\right)^{\alpha-1} f(s) \mathrm{d} s\right\| \\
& \leq \quad \sup _{s \in\left[0, t_{1}-\varepsilon\right]}\left\|\left(t_{2}-s\right)^{1-\alpha} S_{\alpha}\left(t_{2}-s\right)-\left(t_{1}-s\right)^{1-\alpha} S_{\alpha}\left(t_{1}-s\right)\right\| \cdot\|f\|_{L^{p}} b^{\alpha-\frac{1}{p}}\left(\frac{p-1}{\alpha p-1}\right)^{1-\frac{1}{p}} \\
& \quad+2 M\|f\|_{L^{p}} \cdot\left(\frac{p-1}{\alpha p-1}\right)^{1-\frac{1}{p}}\left[\left(t_{2}-t_{1}+\varepsilon\right)^{\frac{\alpha p-1}{p-1}}-\left(t_{2}-t_{1}\right)^{\frac{\alpha p-1}{p-1}}\right]^{1-\frac{1}{p}}
\end{aligned}
$$




$$
\begin{aligned}
& +M\|f\|_{L^{p}}\left(\int_{0}^{t_{1}}\left[\left(t_{2}-s\right)^{\alpha-1}-\left(t_{1}-s\right)^{\alpha-1}\right]^{\frac{p}{p-1}} \mathrm{~d} s\right)^{1-\frac{1}{p}} \\
& +M\|f\|_{L^{p}}\left(\frac{p-1}{\alpha p-1}\right)^{1-\frac{1}{p}}\left(t_{2}-t_{1}\right)^{\alpha-\frac{1}{p}}
\end{aligned}
$$

Then due to the equicontinuity of $\left\{t^{1-\alpha} S_{\alpha}(t), t>0\right\}$, Lemma 2.7 and the arbitrariness of $\varepsilon$, we get

$$
\left\|\left(S_{\alpha} * f\right)\left(t_{2}\right)-\left(S_{\alpha} * f\right)\left(t_{1}\right)\right\| \rightarrow 0, \quad \text { as } t_{1} \rightarrow t_{2},
$$

which shows that $\left(S_{\alpha} * f\right)(t)$ is continuous on $(0, b]$.

Lemma 3.2 Suppose that conditions (H1)-(H3) are satisfied. Then $x \in C_{1-\alpha}(J, X)$ is a solution to problem (1.1) if and only if $x$ satisfies

$$
x(t)=S_{\alpha}(t) x_{0}+\int_{0}^{t} S_{\alpha}(t-s) f(s, x(s), H x(s)) \mathrm{d} s, \quad t \in J^{\prime} .
$$

Proof By Lemma 2.4(b), we know that, for $t>0$,

$$
g_{\alpha}(t)=S_{\alpha}(t)-\left(A g_{\alpha} * S_{\alpha}\right)(t)
$$

Let $x(\cdot)$ be a solution to problem (1.1). Then we have

$$
\begin{aligned}
g_{\alpha} * x & =\left(S_{\alpha}-A g_{\alpha} * S_{\alpha}\right) * x \\
& =S_{\alpha} * x-S_{\alpha} *\left(A g_{\alpha} * x\right) \\
& =S_{\alpha} *\left(x-A g_{\alpha} * x\right) \\
& =S_{\alpha} *\left(g_{\alpha} x_{0}+g_{\alpha} * f(\cdot, x(\cdot), H x(\cdot))\right) \\
& =g_{\alpha} *\left(S_{\alpha} x_{0}+S_{\alpha} * f(\cdot, x(\cdot), H x(\cdot))\right),
\end{aligned}
$$

which implies

$$
x(t)=S_{\alpha}(t) x_{0}+\int_{0}^{t} S_{\alpha}(t-s) f(s, x(s), H x(s)) \mathrm{d} s .
$$

Conversely, suppose $x(\cdot)$ satisfies Eq. (3.2). From Lemma 3.1, we know that $x$ is well defined on $J^{\prime}$. For the result of $A I_{t}^{\alpha} x(t)$, by Definition 2.3(c), we have

$$
\begin{aligned}
\left(s^{1-\alpha} S_{\alpha}(s)-\frac{1}{\Gamma(\alpha)}\right) I_{t}^{\alpha} x(t) \\
=\left(s^{1-\alpha} S_{\alpha}(s)-s^{1-\alpha} g_{\alpha}(s)\right)\left(I_{t}^{\alpha} S_{\alpha}(t) x_{0}+g_{\alpha} * S_{\alpha} * f(\cdot, x(\cdot), H x(\cdot))(t)\right) \\
=s^{1-\alpha}\left[S_{\alpha}(s) I_{t}^{\alpha} S_{\alpha}(t) x_{0}-g_{\alpha}(s) I_{t}^{\alpha} S_{\alpha}(t) x_{0}\right] \\
\quad+s^{1-\alpha}\left[S_{\alpha}(s) \cdot\left(I_{t}^{\alpha} S_{\alpha}\right) * f(\cdot, x(\cdot), H x(\cdot))(t)-g_{\alpha}(s) \cdot\left(I_{t}^{\alpha} S_{\alpha}\right) * f(\cdot, x(\cdot), H x(\cdot))(t)\right] \\
=s^{1-\alpha}\left[S_{\alpha}(t) I_{s}^{\alpha} S_{\alpha}(s) x_{0}-g_{\alpha}(t) I_{s}^{\alpha} S_{\alpha}(s) x_{0}\right]
\end{aligned}
$$




$$
\begin{aligned}
& +s^{1-\alpha}\left[I_{s}^{\alpha} S_{\alpha}(s) S_{\alpha}(t)-I_{s}^{\alpha} S_{\alpha}(s) g_{\alpha}(t)\right] * f(\cdot, x(\cdot), H x(\cdot))(t) \\
= & s^{1-\alpha} I_{s}^{\alpha} S_{\alpha}(s)\left[S_{\alpha}(t) x_{0}-g_{\alpha}(t) x_{0}+S_{\alpha} * f(\cdot, x(\cdot), H x(\cdot))(t)-g_{\alpha} * f(\cdot, x(\cdot), H x(\cdot))(t)\right] \\
= & s^{1-\alpha} I_{s}^{\alpha} S_{\alpha}(s)\left[x(t)-g_{\alpha}(t) x_{0}-I_{t}^{\alpha} f(t, x(t), H x(t))\right] .
\end{aligned}
$$

It follows that

$$
\begin{aligned}
& A I_{t}^{\alpha} x(t) \\
& \quad=\lim _{s \rightarrow 0^{+}} \Gamma(2 \alpha) \frac{\left(s^{1-\alpha} S_{\alpha}(s)-\frac{1}{\Gamma(\alpha)}\right) I_{t}^{\alpha} x(t)}{s^{\alpha}} \\
& \quad=\lim _{s \rightarrow 0^{+}} \Gamma(2 \alpha) s^{1-2 \alpha} I_{s}^{\alpha} S_{\alpha}(s)\left[x(t)-g_{\alpha}(t) x_{0}-I_{t}^{\alpha} f(t, x(t), H x(t))\right] .
\end{aligned}
$$

Noticing that

$$
\begin{aligned}
\| & \Gamma(2 \alpha) s^{1-2 \alpha} I_{s}^{\alpha} S_{\alpha}(s) x-x \| \\
= & \left\|\frac{\Gamma(2 \alpha)}{\Gamma(\alpha)} \int_{0}^{s} s^{1-2 \alpha}(s-\tau)^{\alpha-1} S_{\alpha}(\tau) x \mathrm{~d} \tau-x\right\| \\
= & \left\|\frac{\Gamma(2 \alpha)}{\Gamma(\alpha)} \int_{0}^{1} s^{1-\alpha}(1-\tau)^{\alpha-1} S_{\alpha}(s \tau) x \mathrm{~d} \tau-x\right\| \\
= & \left\|\frac{\Gamma(2 \alpha)}{[\Gamma(\alpha)]^{2}} \int_{0}^{1} s^{1-\alpha} \Gamma(\alpha)(1-\tau)^{\alpha-1} S_{\alpha}(s \tau) x \mathrm{~d} \tau-x\right\| \\
= & \| \frac{\Gamma(2 \alpha)}{[\Gamma(\alpha)]^{2}} \int_{0}^{1}(1-\tau)^{\alpha-1} \tau^{\alpha-1} \Gamma(\alpha)(s \tau)^{1-\alpha} S_{\alpha}(s \tau) x \mathrm{~d} \tau \\
& -\frac{\Gamma(2 \alpha)}{[\Gamma(\alpha)]^{2}} \int_{0}^{1}(1-\tau)^{\alpha-1} \tau^{\alpha-1} x \mathrm{~d} \tau \| \\
\leq & \frac{\Gamma(2 \alpha)}{[\Gamma(\alpha)]^{2}} \int_{0}^{1}(1-\tau)^{\alpha-1} \tau^{\alpha-1} \mathrm{~d} \tau \cdot \sup _{\tau \in[0,1]}\left\|\Gamma(\alpha)(s \tau)^{1-\alpha} S_{\alpha}(s \tau) x-x\right\| \\
\leq & \sup _{\tau \in[0,1]}\left\|\Gamma(\alpha)(s \tau)^{1-\alpha} S_{\alpha}(s \tau) x-x\right\| .
\end{aligned}
$$

By Definition 2.3(a), we get

$$
\left\|\Gamma(2 \alpha) s^{1-2 \alpha} I_{s}^{\alpha} S_{\alpha}(s) x-x\right\| \rightarrow 0, \quad \text { as } s \rightarrow 0^{+} .
$$

Combining (3.3) and (3.4), we have

$$
A I_{t}^{\alpha} x(t)=x(t)-g_{\alpha}(t) x_{0}-I_{t}^{\alpha} f(t, x(t), H x(t)) .
$$

That is,

$$
x(t)=g_{\alpha}(t) x_{0}+A I_{t}^{\alpha} x(t)+I_{t}^{\alpha} f(t, x(t), H x(t)),
$$

which shows that $x$ is a solution to problem (1.1). 
Lemma 3.3 Suppose that assumptions $(\mathrm{H} 1)-(\mathrm{H} 3)$ are satisfied. Let $W_{r}=\left\{x \in C_{1-\alpha}(J, X)\right.$ : $\left.\|x\|_{C_{1-\alpha}} \leq r\right\}$. Then the mapping $G: W_{r} \rightarrow C_{1-\alpha}(J, X)$ defined by

$$
(G x)(t)=\int_{0}^{t} S_{\alpha}(t-s) f(s, x(s), H x(s)) \mathrm{d} s
$$

is compact.

Proof In view of the relationship between $\left(C_{1-\alpha}(J, X),\|\cdot\|_{C_{1-\alpha}}\right)$ and $\left(C(J, X),\|\cdot\|_{C}\right)$, for the compactness of $G W_{r}$ in $C_{1-\alpha}(J, X)$, it is sufficient to prove that the set

$$
B=\left\{y \in C(J, X): y(t)=t^{1-\alpha}(G x)(t), x \in W_{r}, t \in J\right\}
$$

is precompact in $C(J, X)$.

Firstly, we show that $B(t)=\{y(t): y \in B\} \subseteq X$ is precompact in $X$ for every $t \in J$. If $t=0$, then $B(0)=0$ is obviously satisfied. If $t>0$, we can define a set $B^{\varepsilon}(t)=\left\{y^{\varepsilon}(t), x \in W_{r}, t \in\right.$ $\left.J^{\prime}\right\} \subseteq X$, where

$$
y^{\varepsilon}(t)=\varepsilon^{1-\alpha} S_{\alpha}(\varepsilon) \cdot \Gamma(\alpha) t^{1-\alpha} \int_{0}^{t-\varepsilon} S_{\alpha}(t-s-\varepsilon) f(s, x(s), H x(s)) \mathrm{d} s .
$$

For $x \in W_{r}, s \in[0, b]$, we have

$$
\begin{aligned}
\|f(s, x(s), H x(s))\| & \leq \theta(s)+\rho s^{1-\alpha}\left(\|x(s)\|+\left\|\int_{0}^{s} h(s, \tau, x(\tau)) \mathrm{d} \tau\right\|\right) \\
& \leq \theta(s)+\rho s^{1-\alpha}\left(\|x(s)\|+\int_{0}^{s} m\|x(\tau)\| \mathrm{d} \tau\right) \\
& \leq \theta(s)+\rho s^{1-\alpha}\|x(s)\|+\rho s^{1-\alpha} \int_{0}^{s} m \tau^{\alpha-1}\left\|\tau^{1-\alpha} x(\tau)\right\| \mathrm{d} \tau \\
& \leq \theta(s)+\rho r+\rho s^{1-\alpha} m \frac{s^{\alpha}}{\alpha} r \\
& \leq \theta(s)+\rho r+\rho \frac{m s}{\alpha} r \\
& \leq \theta(s)+\rho r+\rho \frac{m b}{\alpha} r .
\end{aligned}
$$

By (3.5), for $x \in W_{r}, t \in(0, b]$, we get

$$
\begin{aligned}
& \left\|t^{1-\alpha} \int_{0}^{t-\varepsilon} S_{\alpha}(t-s-\varepsilon) f(s, x(s), H x(s)) \mathrm{d} s\right\| \\
& \quad \leq b^{1-\alpha} \int_{0}^{t-\varepsilon}\left\|(t-s-\varepsilon)^{1-\alpha} S_{\alpha}(t-s-\varepsilon) \cdot(t-s-\varepsilon)^{\alpha-1} f(s, x(s), H x(s))\right\| \mathrm{d} s \\
& \quad \leq M b^{1-\alpha} \int_{0}^{t-\varepsilon}\left\|(t-s-\varepsilon)^{\alpha-1} f(s, x(s), H x(s))\right\| \mathrm{d} s \\
& \quad \leq M b^{1-\alpha} \int_{0}^{t-\varepsilon}(t-s-\varepsilon)^{\alpha-1}\left(\theta(s)+\rho r+\rho \frac{m b}{\alpha} r\right) \mathrm{d} s
\end{aligned}
$$




$$
\begin{aligned}
& \leq M b^{1-\alpha} \int_{0}^{t-\varepsilon}(t-s-\varepsilon)^{\alpha-1} \theta(s) \mathrm{d} s+M b^{1-\alpha} \int_{0}^{t-\varepsilon}(t-s-\varepsilon)^{\alpha-1}\left(\rho r+\rho \frac{m b}{\alpha} r\right) \mathrm{d} s \\
& \leq M\left(b \frac{p-1}{\alpha p-1}\right)^{1-\frac{1}{p}}\|\theta\|_{L^{p}}+\frac{M b}{\alpha}\left(\rho r+\rho \frac{m b}{\alpha} r\right) \\
& <\infty
\end{aligned}
$$

Moreover, due to hypothesis (H1), for $\varepsilon>0$, the operator $\varepsilon^{1-\alpha} S_{\alpha}(\varepsilon)$ is compact. So we know that $B^{\varepsilon}(t)$ is precompact in $X$ for each $t \in J^{\prime}$.

Let $t \in(0, b]$ and $\delta \in(\varepsilon, t)$. We have

$$
\begin{aligned}
& \left\|y(t)-y^{\varepsilon}(t)\right\| \\
& \leq t^{1-\alpha}\left[\| \int_{0}^{t-\varepsilon}(t-s)^{1-\alpha} S_{\alpha}(t-s) \cdot(t-s)^{\alpha-1} f(s, x(s), H x(s)) \mathrm{d} s\right. \\
& -\varepsilon^{1-\alpha} S_{\alpha}(\varepsilon) \Gamma(\alpha) \int_{0}^{t-\varepsilon}(t-s-\varepsilon)^{1-\alpha} S_{\alpha}(t-s-\varepsilon) \cdot(t-s)^{\alpha-1} f(s, x(s), H x(s)) \mathrm{d} s \| \\
& +\| \varepsilon^{1-\alpha} S_{\alpha}(\varepsilon) \Gamma(\alpha) \int_{0}^{t-\varepsilon}(t-s-\varepsilon)^{1-\alpha} S_{\alpha}(t-s-\varepsilon) \cdot\left((t-s)^{\alpha-1}-(t-s-\varepsilon)^{\alpha-1}\right) \\
& \times f(s, x(s), H x(s)) \mathrm{d} s \| \\
& \left.+\left\|\int_{t-\varepsilon}^{t}(t-s)^{1-\alpha} S_{\alpha}(t-s) \cdot(t-s)^{\alpha-1} f(s, x(s), H x(s)) \mathrm{d} s\right\|\right] \\
& \leq b^{1-\alpha} \int_{0}^{t-\varepsilon} \|\left[(t-s)^{1-\alpha} S_{\alpha}(t-s)-\Gamma(\alpha) \varepsilon^{1-\alpha} S_{\alpha}(\varepsilon)(t-s-\varepsilon)^{1-\alpha} S_{\alpha}(t-s-\varepsilon)\right] \\
& \times(t-s)^{\alpha-1} f(s, x(s), H x(s)) \| \mathrm{d} s \\
& +b^{1-\alpha}\left\|\varepsilon^{1-\alpha} S_{\alpha}(\varepsilon)\right\| \Gamma(\alpha) \cdot M \int_{0}^{t-\varepsilon}\left\|\left[(t-s)^{\alpha-1}-(t-s-\varepsilon)^{\alpha-1}\right] f(s, x(s), H x(s))\right\| \mathrm{d} s \\
& +b^{1-\alpha} M \int_{t-\varepsilon}^{t}\left\|(t-s)^{\alpha-1} f(s, x(s), H x(s))\right\| \mathrm{d} s \\
& \leq b^{1-\alpha} \int_{0}^{t-\delta}\left\|(t-s)^{1-\alpha} S_{\alpha}(t-s)-\Gamma(\alpha) \varepsilon^{1-\alpha} S_{\alpha}(\varepsilon)(t-s-\varepsilon)^{1-\alpha} S_{\alpha}(t-s-\varepsilon)\right\| \\
& \times(t-s)^{\alpha-1}\left(\theta(s)+\rho r+\rho \frac{m b}{\alpha} r\right) \mathrm{d} s \\
& +b^{1-\alpha} \int_{t-\delta}^{t-\varepsilon}\left\|(t-s)^{1-\alpha} S_{\alpha}(t-s)-\Gamma(\alpha) \varepsilon^{1-\alpha} S_{\alpha}(\varepsilon)(t-s-\varepsilon)^{1-\alpha} S_{\alpha}(t-s-\varepsilon)\right\| \\
& \times(t-s)^{\alpha-1}\left(\theta(s)+\rho r+\rho \frac{m b}{\alpha} r\right) \mathrm{d} s \\
& +b^{1-\alpha} M^{2} \Gamma(\alpha)\left(\int_{0}^{t-\varepsilon}\left[(t-s)^{\alpha-1}-(t-s-\varepsilon)^{\alpha-1}\right]^{\frac{p}{p-1}} \mathrm{~d} s\right)^{1-\frac{1}{p}}\|f\|_{L^{p}} \\
& +b^{1-\alpha} M \int_{t-\varepsilon}^{t}\left\|(t-s)^{\alpha-1} f(s, x(s), H x(s))\right\| \mathrm{d} s \\
& :=I_{1}+I_{2}+I_{3}+I_{4} \text {, }
\end{aligned}
$$


where

$$
\begin{aligned}
I_{1}= & b^{1-\alpha} \int_{0}^{t-\delta}\left\|(t-s)^{1-\alpha} S_{\alpha}(t-s)-\Gamma(\alpha) \varepsilon^{1-\alpha} S_{\alpha}(\varepsilon)(t-s-\varepsilon)^{1-\alpha} S_{\alpha}(t-s-\varepsilon)\right\| \\
& \times(t-s)^{\alpha-1}\left(\theta(s)+\rho r+\rho \frac{m b}{\alpha} r\right) \mathrm{d} s, \\
I_{2}= & b^{1-\alpha} \int_{t-\delta}^{t-\varepsilon}\left\|(t-s)^{1-\alpha} S_{\alpha}(t-s)-\Gamma(\alpha) \varepsilon^{1-\alpha} S_{\alpha}(\varepsilon)(t-s-\varepsilon)^{1-\alpha} S_{\alpha}(t-s-\varepsilon)\right\| \\
& \times(t-s)^{\alpha-1}\left(\theta(s)+\rho r+\rho \frac{m b}{\alpha} r\right) \mathrm{d} s, \\
I_{3}= & b^{1-\alpha} M^{2} \Gamma(\alpha)\left(\int_{0}^{t-\varepsilon}\left[(t-s)^{\alpha-1}-(t-s-\varepsilon)^{\alpha-1}\right]^{\frac{p}{p-1}} \mathrm{~d} s\right)^{1-\frac{1}{p}}\|f\|_{L^{p}}, \\
I_{4}= & b^{1-\alpha} M \int_{t-\varepsilon}^{t}\left\|(t-s)^{\alpha-1} f(s, x(s), H x(s))\right\| \mathrm{d} s .
\end{aligned}
$$

From Lemma 2.5, we know that $I_{1} \rightarrow 0$, as $\varepsilon \rightarrow 0^{+}$. By the arbitrariness of $\varepsilon, \delta$ and absolute continuity of integral, we get

$$
I_{2} \rightarrow 0, \quad I_{4} \rightarrow 0,
$$

as $\varepsilon, \delta \rightarrow 0^{+}$. The conclusion of Lemma 2.7 shows that $I_{3} \rightarrow 0$, as $\varepsilon \rightarrow 0^{+}$. Now for $t \in J^{\prime}$, we get

$$
\lim _{\varepsilon \rightarrow 0^{+}}\left\|y(t)-y^{\varepsilon}(t)\right\|=0,
$$

which implies that $B(t)=\{y(t): y \in B\}$ is precompact in $X$ as there is a family of precompact sets arbitrarily close to it.

Next, we show the equicontinuity of $B$ on $J$. Similar to the computational procedure of (3.6), we can get

$$
\begin{aligned}
& \left\|\int_{0}^{t} S_{\alpha}(t-s) f(s, x(s), H x(s)) \mathrm{d} s\right\| \\
& \quad \leq M b^{\alpha-\frac{1}{p}}\left(\frac{p-1}{\alpha p-1}\right)^{1-\frac{1}{p}}\|\theta\|_{L^{p}}+\frac{M b^{\alpha}}{\alpha}\left(\rho r+\rho \frac{m b}{\alpha} r\right) \\
& \quad:=F_{r}<\infty,
\end{aligned}
$$

for $t \in J, x \in W_{r}$. Let $y \in B, 0 \leq t_{1}<t_{2} \leq b$. Then we have

$$
\begin{aligned}
& \left\|y\left(t_{2}\right)-y\left(t_{1}\right)\right\| \\
& =\left\|t_{2}^{1-\alpha} \int_{0}^{t_{2}} S_{\alpha}\left(t_{2}-s\right) f(s, x(s), H x(s)) \mathrm{d} s-t_{1}^{1-\alpha} \int_{0}^{t_{1}} S_{\alpha}\left(t_{1}-s\right) f(s, x(s), H x(s)) \mathrm{d} s\right\| \\
& \leq\left\|\left(t_{2}^{1-\alpha}-t_{1}^{1-\alpha}\right) \int_{0}^{t_{2}} S_{\alpha}\left(t_{2}-s\right) f(s, x(s), H x(s)) \mathrm{d} s\right\| \\
& \quad+t_{1}^{1-\alpha}\left\|\int_{0}^{t_{2}} S_{\alpha}\left(t_{2}-s\right) f(s, x(s), H x(s)) \mathrm{d} s-\int_{0}^{t_{1}} S_{\alpha}\left(t_{1}-s\right) f(s, x(s), H x(s)) \mathrm{d} s\right\|
\end{aligned}
$$




$$
\begin{aligned}
\leq & \left(t_{2}^{1-\alpha}-t_{1}^{1-\alpha}\right) F_{r} \\
& +b^{1-\alpha}\left\|\int_{0}^{t_{2}} S_{\alpha}\left(t_{2}-s\right) f(s, x(s), H x(s)) \mathrm{d} s-\int_{0}^{t_{1}} S_{\alpha}\left(t_{1}-s\right) f(s, x(s), H x(s)) \mathrm{d} s\right\| .
\end{aligned}
$$

From (3.5), we know

$$
\|f(s, x(s), H x(s))\| \leq \theta(s)+\rho r+\rho \frac{m b}{\alpha} r, \quad \theta \in L^{p}(J, X) .
$$

Then due to Eq. (3.1) in Lemma 3.1, we have

$$
\left\|\int_{0}^{t_{2}} S_{\alpha}\left(t_{2}-s\right) f(s, x(s), H x(s)) \mathrm{d} s-\int_{0}^{t_{1}} S_{\alpha}\left(t_{1}-s\right) f(s, x(s), H x(s)) \mathrm{d} s\right\| \rightarrow 0,
$$

as $t_{1} \rightarrow t_{2}$, independent of $x \in W_{r}$. Now we can obtain

$$
\lim _{t_{1} \rightarrow t_{2}}\left\|y\left(t_{2}\right)-y\left(t_{1}\right)\right\|=0
$$

which leads to the equicontinuity of $B$ on $J$. Thus $G: W_{r} \rightarrow C_{1-\alpha}(J, X)$ is a compact mapping by the Ascoli-Arzela theorem. This proof is completed.

Now we can present our main existence result to problem (1.1).

Theorem 3.4 Assume that the hypotheses (H1)-(H3) are satisfied. Then the system (1.1) has at least one solution.

Proof We transform the existence of solutions into a fixed point problem. For this purpose, by considering Lemma 3.2, we introduce the solution operator $\Phi: C_{1-\alpha}(J, X) \rightarrow C_{1-\alpha}(J, X)$ by

$$
\Phi x(t)=S_{\alpha}(t) x_{0}+\int_{0}^{t} S_{\alpha}(t-s) f(s, x(s), H x(s)) \mathrm{d} s
$$

It is easy to see that the fixed point of $\Phi$ is just the solution to problem (1.1). Subsequently, we shall prove that $\Phi$ has a fixed point by Schauder's fixed point theorem.

Step 1. We claim that $\Phi W_{r} \subseteq W_{r}$ in $C_{1-\alpha}(J, X)$, where

$$
r \geq \frac{\alpha^{2}}{\alpha^{2}-M b(\alpha \rho+\rho b m)}\left[M\left\|x_{0}\right\|+M\left(b \frac{p-1}{\alpha p-1}\right)^{1-\frac{1}{p}}\|\theta\|_{L^{p}}\right]
$$

In fact, for $x \in W_{r}, t \in J$, from (3.7) we have

$$
\begin{aligned}
& \left\|t^{1-\alpha} \Phi x(t)\right\| \\
& \quad \leq\left\|t^{1-\alpha} S_{\alpha}(t) x_{0}\right\|+b^{1-\alpha}\left\|\int_{0}^{t} S_{\alpha}(t-s) f(s, x(s), H x(s)) \mathrm{d} s\right\| \\
& \quad \leq M\left\|x_{0}\right\|+M\left(b \frac{p-1}{\alpha p-1}\right)^{1-\frac{1}{p}}\|\theta\|_{L^{p}}+\frac{M b}{\alpha}\left(\rho r+\rho \frac{m b}{\alpha} r\right) \\
& \quad \leq r .
\end{aligned}
$$


Step 2. We show that $\Phi$ is continuous on $W_{r} \subseteq C_{1-\alpha}(J, X)$. For this purpose, we assume that $x_{n} \rightarrow x$ in $W_{r}$. From hypothesis (H2), (H3), for $t \in J$, we have

$$
(t-s)^{\alpha-1}\left(f\left(s, x_{n}(s), H x_{n}(s)\right)-f(s, x(s), H x(s))\right) \rightarrow 0, \quad \text { a.e. } s \in[0, t]
$$

and from (3.5), it follows that

$$
\begin{aligned}
& (t-s)^{\alpha-1}\left\|f\left(s, x_{n}(s), H x_{n}(s)\right)-f(s, x(s), H x(s))\right\| \\
& \quad \leq 2(t-s)^{\alpha-1}\left(\theta(s)+\varrho r+\rho \frac{m b}{\alpha} r\right), \quad s \in[0, t] .
\end{aligned}
$$

Then, by the dominated convergence theorem, we get

$$
\begin{aligned}
& t^{1-\alpha}\left\|\left(\Phi x_{n}\right)(t)-(\Phi x)(t)\right\| \\
& \quad \leq t^{1-\alpha} \int_{0}^{t}\left\|(t-s)^{1-\alpha} S_{\alpha}(t-s)\right\| \cdot(t-s)^{\alpha-1}\left\|f\left(s, x_{n}(s), H x_{n}(s)\right)-f(s, x(s), H x(s))\right\| \mathrm{d} s \\
& \quad \leq M b^{1-\alpha} \int_{0}^{t}(t-s)^{\alpha-1}\left\|f\left(s, x_{n}(s), H x_{n}(s)\right)-f(s, x(s), H x(s))\right\| \mathrm{d} s \\
& \quad \rightarrow 0, \quad n \rightarrow \infty,
\end{aligned}
$$

which implies the continuity of $\Phi$ on $W_{r}$.

Step 3. We show that the operator $\Phi$ is compact. Let

$$
\Phi=\Phi_{1}+\Phi_{2}
$$

where $\Phi_{1}(t)=S_{\alpha}(t) x_{0}, \Phi_{2}(t)=\int_{0}^{t} S_{\alpha}(t-s) f(s, x(s), H x(s)) \mathrm{d} s$. From Lemma 3.3, we have concluded that $\Phi_{2}$ is compact in $W_{r}$. For the compactness of $\Phi_{1}$, it is sufficient to check the set

$$
V=\left\{z \in C(J, X): z(t)=t^{1-\alpha} S_{\alpha}(t) x_{0}, x_{0} \in X, t \in J\right\}
$$

is precompact in $C(J, X)$. Obviously, $V(0)=\left\{\frac{x_{0}}{\Gamma(\alpha)}\right\}, V(t)=\left\{t^{1-\alpha} S_{\alpha}(t) x_{0}\right\}, t>0$, is precompact in $X$. Suppose that $0 \leq t_{1}<t_{2} \leq b$. If $t_{1}=0$, in view of Definition 2.3(a), we get

$$
\left\|z\left(t_{2}\right)-z(0)\right\|=\left\|t_{2}^{1-\alpha} S_{\alpha}\left(t_{2}\right) x_{0}-\frac{x_{0}}{\Gamma(\alpha)}\right\| \rightarrow 0
$$

as $t_{2} \rightarrow 0$. If $t_{1}>0$,

$$
\left\|z\left(t_{2}\right)-z\left(t_{1}\right)\right\| \leq\left\|t_{2}^{1-\alpha} S_{\alpha}\left(t_{2}\right) x_{0}-t_{2}^{1-\alpha} S_{\alpha}\left(t_{2}\right) x_{0}\right\| \rightarrow 0
$$

From hypothesis (H1), we know that $\left\|z\left(t_{2}\right)-z\left(t_{1}\right)\right\| \rightarrow 0$, as $t_{1} \rightarrow t_{2}$. By the Ascoli-Arzela theorem, we see that $V$ is precompact in $C(J, X)$. Therefore, $\Phi=\Phi_{1}+\Phi_{2}$ is a compact operator in $C_{1-\alpha}(J, X)$.

Hence, from Schauder's fixed point theorem, there exists a fixed point $x$ such that $\Phi x=x$, which is the solution to problem (1.1). This completes the proof. 


\section{Application}

Consider the following integrodifferential evolution system with Riemann-Liouville fractional derivative:

$$
\left\{\begin{array}{l}
D^{\alpha} u(t, x)=\frac{\partial^{2}}{\partial x^{2}} u(t, x)+F\left(t, u(t, x), \int_{0}^{t} h_{1}(t, s, u(s, x)) \mathrm{d} s\right), \quad 0<t \leq 1,0<x<1 \\
u(t, 0)=u(t, 1)=0 \\
\lim _{t \rightarrow 0^{+}} \Gamma(\alpha) t^{1-\alpha} u(t, x)=u_{0}(x) .
\end{array}\right.
$$

Take $X=L^{2}(0,1)$ and the operator $A: D(A) \subseteq X \rightarrow X$ defined by $A z=z^{\prime \prime}$, with

$$
D(A)=\left\{z \in X: z, z^{\prime} \text { are absolutely continuous, } z^{\prime \prime} \in X, z(0)=z(1)=0\right\} .
$$

From Pazy [36], $A$ is the infinitesimal generator of a compact analytic semigroup $T(t)$, $t \geq 0$. It is known that $A$ has the eigenvalues $\lambda_{n}=-n^{2} \pi^{2}, n \in \mathbb{N}$, and the corresponding eigenvectors $e_{n}(x)=\sqrt{2} \sin (n \pi x)$ for $n \geq 1, e_{0}=1$, which form an orthogonal basis for $L^{2}(0,1)$. Then $T(t)$ is given by

$$
T(t) z=\sum_{n=1}^{\infty} e^{-n^{2} \pi^{2} t}\left\langle z, e_{n}\right\rangle e_{n}
$$

If $u_{0}(x)=\sum_{n=1}^{\infty} c_{n} \sin n \pi x$, then we have

$$
T(t) u_{0}(x)=\sum_{n=1}^{\infty} e^{-n^{2} \pi^{2} t} c_{n} \sin n \pi x
$$

Moreover, from [21], we know $A$ is the infinitesimal generator of an order- $\alpha$ fractional resolvent $S_{\alpha}(t)$ and

$$
S_{\alpha}(t) u_{0}(x)=\sum_{n=1}^{\infty} t^{\alpha-1} E_{\alpha, \alpha}\left(-n^{2} \pi^{2} t^{\alpha}\right) c_{n} \sin n \pi x
$$

Employing the method in $[13,31]$, by Laplace transformation and probability density functions, we can have

$$
t^{1-\alpha} S_{\alpha}(t) u_{0}(x)=\alpha \int_{0}^{\infty} \theta \xi_{\alpha}(\theta) T\left(t^{\alpha} \theta\right) u_{0}(x) \mathrm{d} \theta
$$

for any $u_{0} \in X$, where

$$
\begin{aligned}
& \xi_{\alpha}(\theta)=\frac{1}{\alpha} \theta^{-1-\frac{1}{\alpha}} \varpi_{\alpha}\left(\theta^{-\frac{1}{\alpha}}\right), \\
& \varpi_{\alpha}(\theta)=\frac{1}{\pi} \sum_{n=1}^{\infty}(-1)^{n-1} \theta^{-n \alpha-1} \frac{\Gamma(n \alpha+1)}{n !} \sin (n \pi \alpha), \quad \theta \in(0, \infty) .
\end{aligned}
$$

Equation (4.2) shows

$$
t^{1-\alpha} S_{\alpha}(t)=\alpha \int_{0}^{\infty} \theta \xi_{\alpha}(\theta) T\left(t^{\alpha} \theta\right) \mathrm{d} \theta
$$


From Lemma 2.9 of [13], it follows that the family of operators $\left\{t^{1-\alpha} S_{\alpha}(t): t>0\right\}$ is equicontinuous, compact and $\left\|t^{1-\alpha} S_{\alpha}(t)\right\| \leq \frac{\alpha M^{\prime}}{\Gamma(1+\alpha)}:=M$, where $M^{\prime}=\sup \{\|T(t)\|: 0 \leq t \leq 1\}$. Then hypothesis (H1) is satisfied.

Now, we define a continuous function $f:[0, b] \times X \times X \rightarrow X$ by

$$
\begin{aligned}
& f(t, u, h)(x)=F(t, u(t, x), h(t, x)), \quad 0<t \leq 1,0<x<1, \\
& h(t, x)=\int_{0}^{t} h_{1}(t, s, u(s, x)) \mathrm{d} s .
\end{aligned}
$$

We take

$$
\begin{aligned}
& F\left(t, u(t, x), \int_{0}^{t} h_{1}(t, s, u(s, x)) \mathrm{d} s\right) \\
& \quad=e^{-t} \cos (u(t, x))+\rho t^{1-\alpha}\left(u(t, x)+\int_{0}^{t} \cos (t s) u(s, x) \mathrm{d} s\right),
\end{aligned}
$$

where $0<\rho<\frac{\alpha^{2}}{M \alpha+M}$. So the functions $f, h$ satisfy hypotheses (H2) and (H3). Let $u(t) x=$ $u(t, x)$, for $t, x \in(0,1)$. Then the differential system (4.1) can be presented in the abstract form (1.1) and all the conditions of Theorem 3.4 are satisfied. Hence there exists a function $u \in C_{1-\alpha}\left(J, L^{2}(0,1)\right)$ which is a solution of $(4.1)$.

\section{Conclusions}

By using fractional resolvents, this paper introduces the solution to semilinear RiemannLiouville fractional integrodifferential equations and discuss its existence results. There are two points worth of attention in the study. One is that the Riemann-Liouville fractional resolvent $S_{\alpha}(t)$ is not bounded at $t=0$, which is essentially different from the case for Caputo fractional resolvents, and the other is $S_{\alpha}(t)$ does not have the property of semigroups, which means that the compactness of $S_{\alpha}(t)$ (or $t^{1-\alpha} S_{\alpha}(t)$ ) cannot conclude the equicontinuity of $S_{\alpha}(t)$ (or $t^{1-\alpha} S_{\alpha}(t)$ ). As the existence of solutions is the basis of qualitative study to differential equations, we can continue to discuss the controllability and stability of the solution using the similar approach. On the other hand, some new general fractional derivatives are introduced and studied, such as some types of extended Riemann-Liouville fractional derivative and the fractional derivative without singular kernel of exponential function [37-41]. Especially, the Hilfer fractional derivative is often used as a generalized Riemann-Liouville fractional derivative, which includes Riemann-Liouville and Caputo derivatives; see $[4,9,42]$. To the best of our knowledge, most of the existence and controllability results on the Hilfer fractional differential system are studied under the frame that $A$ generates a strongly continuous semigroup and the solution is given by semigroup and probability density functions. It is still an open problem how to define fractional resolvents to Hilfer and other general fractional equations and it is worth discussing later.

\section{Acknowledgements}

This work was completed when the first author visited Texas A\&M University. The author is grateful to Professor Goong Chen and Department of Mathematics for their hospitality and providing good working conditions. The authors are grateful to the referees for their careful reading of the manuscript and their valuable comments. 
Availability of data and materials

Data sharing is not applicable to this article as no data sets were generated or analyzed during the current study.

\section{Competing interests}

The authors declare that they have no competing interests.

\section{Authors' contributions}

The work presented in this paper has been accomplished through contributions of all authors. All authors read and approved the final manuscript.

\section{Author details}

${ }^{1}$ Faculty of Mathematics and Physics, Huaiyin Institute of Technology, Huaian, P.R. China. ${ }^{2}$ School of Mathematical Science, Huaiyin Normal University, Huaian, P.R. China.

\section{Publisher's Note}

Springer Nature remains neutral with regard to jurisdictional claims in published maps and institutional affiliations.

Received: 1 August 2019 Accepted: 10 December 2019 Published online: 18 December 2019

\section{References}

1. Bonilla, B., Rivero, M., Rodriguez-Germa, L., Trujillo, J.J.: Fractional differential equations as alternative models to nonlinear differential equations. Appl. Math. Comput. 187, 79-88 (2007)

2. Miller, K.S., Ross, B.: An Introduction to the Fractional Calculus and Differential Equations. Wiley, New York (1993)

3. Podlubny, I.: Fractional Differential Equations. Academic Press, San Diego (1999)

4. Hilfer, R:: Applications of Fractional Calculus in Physics. World Scientific, Singapore (2000)

5. Kilbas, A.A., Srivastava, H.M., Trujillo, J.J.: Theory and Applications of Fractional Differential Equations. Elsevier, Amsterdam (2006)

6. Lakshmikantham, V., Leela, S., Vasundhara Devi, J.: Theory of Fractional Dynamic Systems. Cambridge Academic Publishers, Cambridge (2009)

7. Yang, X.J.: General Fractional Derivatives: Theory, Methods and Applications. CRC Press, New York (2019)

8. El-Borai, M.M.: Some probability densities and fundamental solutions of fractional evolution equations. Chaos Solitons Fractals 14, 433-440 (2002)

9. Du, J., Jiang, W., Pang, D., Niazi, A.U.K.: Exact controllability for Hilfer fractional differential inclusions involving nonlocal initial conditions. Complexity 2018, 9472847 (2018)

10. Li, M., Chen, C., Li, F.B.: On fractional powers of generators of fractional resolvent families. J. Funct. Anal. 259, 2702-2726 (2010)

11. Balachandran, K., Kiruthika, S.: Existence results for fractional integrodifferential equations with nonlocal condition via resolvent operators. Comput. Math. Appl. 62, 1350-1358 (2011)

12. Hernandez, E., O'Regan, D., Balachandran, K.: Existence results for abstract fractional differential equations with nonlocal conditions via resolvent operators. Indag. Math. 24, 68-82 (2013)

13. Wang, J., Zhou, Y.: A class of fractional evolution equations and optimal controls. Nonlinear Anal., Real World Appl. 12 262-272 (2011)

14. Wang, J., Fečkan, M., Zhou, Y.: Approximate controllability of Sobolev type fractional evolution systems with nonlocal conditions. Evol. Equ. Control Theory 6, 471-486 (2017)

15. Wang, J., Zhou, Y., Wei, W., Xu, H.: Nonlocal problems for fractional integrodifferential equations via fractional operators and optimal controls. Comput. Math. Appl. 62, 1427-1441 (2011)

16. Fan, Z:: Characterization of compactness for resolvents and its applications. Appl. Math. Comput. 232, 60-67 (2014)

17. Ji, S.: Approximate controllability of semilinear nonlocal fractional differential systems via an approximating method. Appl. Math. Comput. 236, 43-53 (2014)

18. Lian, T., Fan, Z., Li, G.: Approximate controllability of semilinear fractional differential systems of order $1<q<2$ via resolvent operators. Filomat 31, 5769-5781 (2017)

19. Agarwal, R.P., Santos, J.P., Cuevas, C.: Analytic resolvent operator and existence results for fractional integrodifferential equations. J. Abstr. Differ. Equ. Appl. 2(2), 26-47 (2012)

20. Chen, L., Fan, Z., Li, G.: On a nonlocal problem for fractional differential equations via resolvent operators. Adv. Differ. Equ. 2014, 251 (2014)

21. Li, K., Peng, J.: Fractional resolvents and fractional evolution equations. Appl. Math. Lett. 25, 808-812 (2012)

22. Li, K., Peng, J., Jia, J.: Cauchy problems for fractional differential equations with Riemann-Liouville fractional derivatives. J. Funct. Anal. 263, 476-510 (2012)

23. Balasubramaniam, P., Tamilalagan, P.: The solvability and optimal controls for impulsive fractional stochastic integro-differential equations via resolvent operators. J. Optim. Theory Appl. 174, 139-155 (2017)

24. Bajlekova, E.G.: Fractional evolution equations in Banach spaces. Ph.D. thesis, Eindhoven University of Technology (2001)

25. Debbouche, A., Baleanu, D.: Controllability of fractional evolution nonlocal impulsive quasilinear delay integro-differential systems. Comput. Math. Appl. 62, 1442-1450 (2011)

26. Mallika, D., Baleanu, D., Suganya, S., Arjunan, M.M.: Existence results for fractional neutral integro-differential systems with nonlocal condition through resolvent operators. An. Ştiinţ. Univ. 'Ovidius' Constanţa, Ser. Mat. 27, 107-124 (2019)

27. Tariboon, J., Ntouyas, S.K., Agarwal, P.: New concepts of fractional quantum calculus and applications to impulsive fractional q-difference equations. Adv. Differ. Equ. 2015, 18 (2015)

28. Heymans, N., Podlubny, I.: Physical interpretation of initial conditions for fractional differential equations with Riemann-Liouville fractional derivatives. Rheol. Acta 45, 765-771 (2006) 
29. Agarwal, R.P., Belmekki, M., Benchohra, M.: A survey on semilinear differential equations and inclusions involving Riemann-Liouville fractional derivative. Adv. Differ. Equ. 2009, 981728 (2009)

30. Baleanu, D., Agarwal, P., Parmar, R.K., et al.: Extension of the fractional derivative operator of the Riemann-Liouville J. Nonlinear Sci. Appl. 10, 2914-2924 (2017)

31. Liu, Z., Li, X.: Approximate controllability of fractional evolution systems with Riemann-Liouville fractional derivatives. SIAM J. Control Optim. 53, 1920-1933 (2015)

32. Lizama, C.: An operator theoretical approach to a class of fractional order differential equations. Appl. Math. Lett. 24, 184-190 (2011)

33. Fan, Z:: Existence and regularity of solutions for evolution equations with Riemann-Liouville fractional derivatives Indag. Math. 25, 516-524 (2014)

34. Zeidler, E.: Nonlinear Functional Analysis and Its Application II/A. Springer, New York (1990)

35. Arendt, W., Batty, C., Hieber, M., Neubrander, F.: Vector-valued Laplace transforms and Cauchy problems. In: Amann, H., Bourguignon, J.P., Grove, K., Lions, P.L. (eds.) Monographs in Mathematics, 2nd edn. vol. 96. Birkhäuser, Basel (2011)

36. Pazy, A.: Semigroups of Linear Operators and Applications to Partial Differential Equations. Springer, New York (1983)

37. Agarwal, P., Choi, J., Paris, R.B.: Extended Riemann-Liouville fractional derivative operator and its applications. J. Nonlinear Sci. Appl. 8, 451-466 (2015)

38. Agarwal, P., Nieto, J.J., Luo, M.J.: Extended Riemann-Liouville type fractional derivative operator with applications Open Math. 15, 1667-1681 (2017)

39. Yang, X.J., Srivastava, H.M., Tenreiro Machado, J.A.: A new fractional derivative without singular kernel: application to the modelling of the steady heat flow. Therm. Sci. 20, 753-756 (2016)

40. Yang, X.J., Gao, F., Tenreiro Machado, J.A., Baleanu, D.: A new fractional derivative involving the normalized sinc function without singular kernel. Eur. Phys. J. Spec. Top. 226, 3567-3575 (2017)

41. Yang, X.J., Gao, F., Ju, Y., Zhou, H.W.: Fundamental solutions of the general fractional-order diffusion equations. Math Methods Appl. Sci. 41, 9312-9320 (2018)

42. Ahmed, H.M., El-Borai, M.M.: Hilfer fractional stochastic integro-differential equations. Appl. Math. Comput. 331, 182-189 (2018)

\section{Submit your manuscript to a SpringerOpen ${ }^{\circ}$ journal and benefit from:}

- Convenient online submission

- Rigorous peer review

- Open access: articles freely available online

- High visibility within the field

- Retaining the copyright to your article

Submit your next manuscript at $\boldsymbol{s p r i n g e r o p e n . c o m ~}$ 